\title{
On the Distribution of Nonlinear Congruential Pseudorandom Numbers of Higher Orders in Residue Rings
}

\author{
Edwin D. El-Mahassni ${ }^{1}$ and Domingo Gomez ${ }^{2, \star}$ \\ 1 Department of Computing, Macquarie University \\ North Ryde, NSW 2109, Australia \\ edwinelm@ics.mq.edu.au \\ 2 Faculty of Science, University of Cantabria \\ E-39071 Santander, Spain \\ domingo.gomez@unican.es
}

\begin{abstract}
The nonlinear congruential method is an attractive alternative to the classical linear congruential method for pseudorandom number generation. In this paper we present new discrepancy bounds for sequences of $s$-tuples of successive nonlinear congruential pseudorandom numbers of higher orders modulo a composite integer $M$.
\end{abstract}

\section{Background}

For an integer $M>1$, we denote by $\mathbb{Z}_{M}$ the residue ring modulo $M$. In this paper we present some distribution properties of a generalization of pseudorandom number generators, first introduced in 7], defined by a recurrence

$$
u_{n+1} \equiv f\left(g_{1}\left(u_{n}, \ldots, u_{n-r+1}\right), \ldots, g_{r}\left(u_{n}, \ldots, u_{n-r+1}\right)\right) \quad(\bmod M),
$$

where

$$
f\left(X_{1}, \ldots, X_{r}\right), g_{1}\left(X_{1}, \ldots, X_{r}\right), \ldots, g_{r}\left(X_{1}, \ldots, X_{r}\right) \in \mathbb{Z}_{M}\left[X_{1}, \ldots, X_{r}\right]
$$

for $n \geq r-1$ with some initial values $u_{0}, \ldots, u_{r-1}$.

To study this pseudorandom number generator, we define the sequence of polynomials $f_{k}(\mathbf{X}) \in \mathbb{Z}_{M}[\mathbf{X}]$, with $\mathbf{X}=X_{1}, \ldots, X_{r}$ by the recurrence relation

$$
f_{k}(\mathbf{X}) \equiv f_{k-1}\left(g_{1}(\mathbf{X}), \ldots, g_{r}(\mathbf{X})\right) \quad(\bmod M), \quad k \geq 1
$$

where $f_{0}(\mathbf{X})=f(\mathbf{X})$.

It is obvious that (10) becomes periodic with some period $t \leq M^{r}$. Throughout this paper we assume that this sequence is purely periodic, i.e. $u_{n}=u_{n+t}$ beginning with $n=0$, otherwise we consider a shift of the original sequence.

* Domingo Gomez is partially supported by the Spanish Ministry of Education and Science grant MTM20067088.

M. Bras-Amorós and T. Høholdt (Eds.): AAECC 2009, LNCS 5527, pp. 195 2032009.

(C) Springer-Verlag Berlin Heidelberg 2009 
Although the distribution of nonlinear congruential generators has been studied extensively, see [5]69 for instance, much less is known for its higher orders analogue. A result for a class of polynomials for prime moduli was established in [7, where this was later extended to a larger family of polynomials in 8. In this paper we show a generalization of this result to a larger class of pseudorandom number generators.

\subsection{Notation and First Results}

This section begins with some notation. It will be assumed that $N, A_{i}, B_{i}$ and $b_{i}$ represent integer positive numbers and $\mathbf{0}$ is the $r$-dimensional 0 vector. The elements of $\mathbb{Z}_{M}$ will be identified with the integers $\{0, \ldots, M-1\}$. For this reason, we can define $\mathbf{e}_{M}(z)=\exp (2 \pi i z / M)$ for any element $z \in \mathbb{Z}_{M}$.

For a polynomial $f, G$ is the gcd of the coefficients of nonconstant monomials with $M$.

We will denote $f^{(p)}(\mathbf{X}) \equiv f(\mathbf{X}) \quad(\bmod p)$ of total degree $d^{\prime}$ for a polynomial $f$ with integer coefficients and total degree $d$. Finally, we define $\operatorname{deg}_{X_{r}} f$, the degree of the coefficient $X_{r}$ of the polynomial $f$, to be $\operatorname{deg}_{X_{r}}$ modulo every prime factor $p$ of $M$.

Lemma 1. Given a polynomial in the ring $\mathbb{Z}_{M}[\mathbf{X}]$,

$$
f(\mathbf{X}) \equiv \sum_{i_{1}=0}^{d_{1}} \ldots \sum_{i_{r}=0}^{d_{r}} b_{i_{1}, \ldots, i_{r}} X_{1}^{i_{1}} \ldots X_{r}^{i_{r}} \quad(\bmod M)
$$

with $\operatorname{deg}_{X_{r}} f \geq 1$, total degree $d$, then exist polynomials

$h_{1}\left(X_{r}\right), \ldots, h_{r-1}\left(X_{r}\right)$ of degree less than $d(\lceil\log d\rceil+1)$ such that

$$
g^{(p)}\left(X_{r}\right)=f^{(p)}\left(a_{1}+h_{1}^{(p)}\left(X_{r}\right), \ldots, a_{r-1}+h_{r-1}^{(p)}\left(X_{r}\right), X_{r}\right)
$$

is a nonconstant polynomial for any $p \mid M, p \nmid G$ and any values $a_{1}, \ldots, a_{r-1} \in \mathbb{Z}_{M}$.

Proof. Let $p \mid M$ be a prime number not dividing $G$ and $D=\lceil\log d\rceil+1$. By the definition of $G$, we note that $f^{(p)}(\mathbf{X})$ is not a constant polynomial and it can be expressed as $f^{(p)}(\mathbf{X})=h\left(X_{1}, \ldots, X_{r-1}\right) X_{r}^{d^{\prime}}+f^{\prime}(\mathbf{X})$ where $f^{\prime}(\mathbf{X})$ is a polynomial of degree stricly less than $d^{\prime}$ in $X_{r}$. If $d^{\prime}=0$ then $f^{(p)}(\mathbf{X})=h\left(X_{1}, \ldots, X_{r-1}\right)$, but in any case $h$ is a not a constant polynomial.

Let $\mathbb{F}$ be an extension field of degree $D$ over $\mathbb{Z}_{p}$. By the cardinality of $\mathbb{F}$, there exist $\xi_{1}, \ldots, \xi_{r-1} \in \mathbb{F}$ such as $h\left(\xi_{1}, \ldots, \xi_{r-1}\right) \neq 0$.

It is easy to check that

$$
f^{(p)}\left(X_{1}+\xi_{1} X_{r}, \ldots, X_{r-1}+\xi_{r-1} X_{r}, X_{r}\right)=h\left(\xi_{1}, \ldots, \xi_{r-1}\right) X_{r}^{d^{\prime \prime}}+f^{\prime \prime}(\mathbf{X})
$$

where $d \geq d^{\prime \prime}>0$ and $f^{\prime \prime}(\mathbf{X})$ is a polynomial with total degree less than $d^{\prime \prime}-1$ in $X_{r}$. Let $\mathbf{E}$ be a extension field of degree $d+1$ over $\mathbb{F}$ and let $\theta$ be a defining element of $\mathbf{E}$ over both $\mathbb{Z}_{p}$ and $\mathbf{E}$, i.e. $\mathbf{E} \equiv \mathbb{Z}_{p}(\theta) \equiv \mathbb{F}(\theta)$. 
The evaluation of the polynomial in (4)

$$
f^{(p)}\left(a_{1}+\xi_{1} \theta, \ldots, a_{r-1}+\xi_{r-1} \theta, \theta\right) \neq 0, \quad a_{1}, \ldots, a_{r-1} \in \mathbb{Z}_{p}
$$

because the degree of the minimal polynomial of $\theta$ over $\mathbb{F}$ is $d+1$.

For $i=1, \ldots, r-1$, each element $\xi_{i} \theta$ can be expressed as $h_{i}^{(p)}(\theta)$, where $h_{i}^{(p)}\left(X_{r}\right) \in$ $\mathbb{Z}_{p}\left[X_{r}\right]$. Applying the Chinese Remainder Theorem to the different polynomials $h_{i}^{(p)}\left(X_{r}\right)$ for each prime $p \mid M$, we find the corresponding $h_{i}\left(X_{r}\right)$. By construction, $f^{(p)}\left(a_{1}+h_{1}^{(p)}\left(X_{r}\right), \ldots, a_{r-1}+h_{r-1}^{(p)}\left(X_{r}\right), X_{r}\right)$ is not the zero polynomial by (5) for any integer values $a_{1}, \ldots, a_{r-1}$.

Now, we proceed to define a family of polynomials depending on $g_{1}(\mathbf{X}), \ldots, g_{r}(\mathbf{X})$ which will be the main subject of the article.

Let $\mathbb{K}$ be a field. We denote by $\mathcal{T}$ as the set of polynomials, $f$, such that $\sum_{j=0}^{s-1} a_{j}\left(f_{k+j}(\mathbf{X})-f_{l+j}(\mathbf{X})\right)$ is nonconstant, where $f_{i}(\mathbf{X})$ are defined by (2) and $a_{j} \in \mathbb{K}$, with at least one $a_{j} \neq 0$ and $k \neq l$.

Here is a sufficient condition for a certain polynomial to be in class $\mathcal{T}$. To prove the result, we need some background.

We start defining a homomorphism of polynomial rings $\phi: \mathbb{K}\left[X_{1}, \ldots, X_{r}\right] \rightarrow$ $\mathbb{K}\left[X_{1}, \ldots, X_{r}\right]$ with $\phi\left(X_{i}\right)=g_{i}(\mathbf{X})$.

Polynomials $g_{1}(\mathbf{X}), \ldots, g_{r}(\mathbf{X})$ are said to be algebraically independent if the application $\phi$ is injective. $\phi^{k}$ denotes the composition of the function $\phi k$ times with $\phi^{0}$ being the identity map.

Lemma 2. Let $f(\mathbf{X})$ be a polynomial in $\mathbb{K}[\mathbf{X}]$ and $g_{1}(\mathbf{X}), \ldots, g_{r}(\mathbf{X})$ be algebraically independent and $\mathbb{F}$ be an extension field of $\mathbb{K}$. Suppose that there exists $\left(b_{1}, \ldots, b_{r}\right),\left(c_{1}, \ldots, c_{r}\right) \in \mathbb{F}^{r}$ two different zeros of the polynomials $g_{1}(\mathbf{X}), \ldots, g_{r}(\mathbf{X})$ with $f\left(c_{1}, \ldots, c_{r}\right) \neq f\left(b_{1}, \ldots, b_{r}\right)$, then $f \in \mathcal{T}$.

Proof. Suppose that $k>l$, and exist $a_{0}, \ldots, a_{s-1} \in \mathbb{K}$ with $a_{0} \neq 0$ satisfying; $\sum_{j=0}^{s-1} a_{j}\left(f_{k+j}(\mathbf{X})-f_{l+j}(\mathbf{X})\right)=K$, where $K \in \mathbb{K}$.

Then

$$
\sum_{j=0}^{s-1} a_{j}\left(f_{k+j}(\mathbf{X})-f_{l+j}(\mathbf{X})\right)=\phi^{k-1}\left(\sum_{j=0}^{s-1} a_{j}\left(f_{1+j}(\mathbf{X})-f_{1-k+l+j}(\mathbf{X})\right)\right)
$$

and this implies $K=\sum_{j=0}^{s-1} a_{j}\left(\left(f_{1+j}(\mathbf{X})\right)-\left(f_{1-k+l+j}(\mathbf{X})\right)\right)$ because $\phi$ is an injective map.

By equation (2), we notice that for $k \neq 0$, we have that $f_{k}\left(b_{1}, \ldots, b_{r}\right)=$ $f_{k-1}(0, \ldots, 0)=f_{k}\left(c_{1}, \ldots, c_{r}\right)$, so substituting in the equation both points and subtracting the result, we get that $a_{0}=0$.

The last remark in this section is that conditions in this criterion can be tested using Groebner basis. 


\subsection{Exponential Sums and Previous Results}

We start by listing some previous bounds on exponential sums which will be used to establish our main results.

The first Lemma is the well-known Hua-Loo Keng bound in a form which is a relaxation of the main result of [1] (see also Section 3 of [3] and Lemma 2.2 in [6]), followed by its multidimensional version.

Lemma 3. For any polynomial $f(X)=b_{d} X^{d}+\ldots+b_{1} X+b_{0} \in \mathbb{Z}_{M}[X]$ of degree $d \geq 1$, there is a constant $c_{0}>0$ where the bound

$$
\left|\sum_{x \in \mathbb{Z}_{M}} \mathbf{e}_{M}(f(x))\right|<e^{c_{0} d} M^{1-1 / d} G^{1 / d}
$$

holds, where $G=\operatorname{gcd}\left(b_{d}, \ldots, b_{1}, M\right)$.

Lemma 4. Let $f(\mathbf{X})$, with total degree $d \geq 2$ and degree greater than one in $X_{r}$, be a polynomial with integer coefficients, with $G=1$. Then the bound

$$
\left|\sum_{x_{1}, \ldots, x_{r} \in \mathbb{Z}_{M}} \mathbf{e}_{M}\left(f\left(x_{1}, \ldots, x_{r}\right)\right)\right| \leq e^{c_{0} d^{2}(\log d+1)} M^{r-1 /\left(d^{2}(\log d+1)\right)}
$$

holds, where $c_{0}$ is some positive constant.

Proof. We recall the univariate case that appears as Lemma 3 Then let

$$
g(\mathbf{X})=f\left(X_{1}+h_{1}\left(X_{r}\right), X_{2}+h_{2}\left(X_{r}\right), \ldots, X_{r}\right)
$$

where $h_{i}\left(X_{r}\right)$ are the polynomials defined in Equation (3). It is easy to see that

$$
\left|\sum \mathbf{e}_{M}\left(f\left(x_{1}, x_{2} \ldots, x_{r}\right)\right)\right|=\left|\sum \mathbf{e}_{M}\left(g\left(x_{1}, x_{2} \ldots, x_{r}\right)\right)\right| .
$$

where the summations are taken over $x_{1}, x_{2} \ldots, x_{r} \in \mathbb{Z}_{M}$ since $\left(x_{1}, \ldots, x_{r}\right) \rightarrow$ $\left(x_{1}+h_{1}\left(x_{r}\right), x_{2}+h_{2}\left(x_{r}\right), \ldots, x_{r}\right)$ merely permutates the points. By Lemma 1 . for any selection $x_{1}, \ldots, x_{r-1}$ this polynomial is not constant modulo $p$ and the gcd of the coefficients of $g$ and $M$ are coprime. Hence, applying Lemma 3, we have

$$
\begin{aligned}
\left|\sum_{x_{1}, x_{2} \ldots, x_{r} \in \mathbb{Z}_{M}} \mathbf{e}_{M}\left(g\left(x_{1}, x_{2} \ldots, x_{r}\right)\right)\right| & \\
& \leq \sum_{x_{1}, \ldots, x_{r-1} \in \mathbb{Z}_{M}}\left|\sum_{x_{r} \in \mathbb{Z}_{M}} \mathbf{e}_{M}\left(g\left(x_{1}, x_{2} \ldots, x_{r}\right)\right)\right| \\
& \leq e^{c_{0} d^{2}(\log d+1)} M^{r-1 / d^{2}(\log d+1)} .
\end{aligned}
$$

We obtain the last step by noting that the degree of $g$ in $X_{r}$ can be bounded by $d^{2}(\log d+1)$ and so we are done. 
This now allows us to state and prove the following Lemma.

Lemma 5. Let $f(\mathbf{X})$ be a polynomial with integer coefficients with $\operatorname{deg}_{X_{r}} f \geq 1$ and total degree $d$. Recalling the definition of $G$,

$$
\left|\sum_{x_{1} \ldots, x_{r} \in \mathbb{Z}_{M}} \mathbf{e}_{M}\left(f\left(x_{1}, \ldots, x_{r}\right)\right)\right| \leq e^{c_{0} d^{2}(\log d+1)} M^{r}(G / M)^{1 / d^{2}(\log d+1)}
$$

Proof. We let

$$
f_{G}\left(x_{1}, \ldots, x_{r}\right)=\left(f\left(x_{1}, \ldots, x_{r}\right)-f(\mathbf{0})\right) / G
$$

and $m=M / G$.

Then,

$$
\begin{aligned}
\left|\sum_{x_{1}, \ldots, x_{r} \in \mathbb{Z}_{M}} \mathbf{e}_{M}\left(f\left(x_{1}, \ldots, x_{r}\right)\right)\right| & =\left|\sum_{x_{1}, \ldots, x_{r} \in \mathbb{Z}_{M}} \mathbf{e}_{M}\left(f\left(x_{1}, \ldots, x_{r}\right)-f(\mathbf{0})\right)\right| \\
& =G^{r}\left|\sum_{x_{1}, \ldots, x_{r} \in \mathbb{Z}_{m}} \mathbf{e}_{m}\left(f_{G}\left(x_{1}, \ldots, x_{r}\right)\right)\right|
\end{aligned}
$$

Now $f_{G}\left(x_{1}, \ldots, x_{r}\right)$ satisfies the conditions in Lemma 4, so:

$$
G^{r}\left|\sum_{x_{1}, \ldots, x_{r} \in \mathbb{Z}_{m}} \mathbf{e}_{m}\left(f_{G}\left(x_{1}, \ldots, x_{r}\right)\right)\right| \leq G^{r} e^{c_{0} d^{2}(\log d+1)}(m)^{r-1 / d^{2}(\log d+1)}
$$

and so the result follows.

Lastly, we will make use of the following lemma, which is essentially the multidimensional version of Lemma 2.3 of $\underline{6}$.

Lemma 6. Let $f(\mathbf{X}) \in \mathbb{Z}_{M}[\mathbf{X}]$ be a polynomial such that $f^{(p)} \in \mathcal{T}$ for every $p \mid M$ and let

$$
\sum_{j=0}^{s-1} a_{j}\left(f_{k+j}(\mathbf{X})-f_{l+j}(\mathbf{X})\right)=\sum_{i_{1}=0}^{d_{1}} \ldots \sum_{i_{r}=0}^{d_{r}} b_{i_{1}, \ldots, i_{r}} X_{1}^{i_{1}} \ldots X_{r}^{i_{r}},
$$

where $k \neq l$. Recalling the definition of $G$, the following equality $G=\operatorname{gcd}\left(a_{0}, \ldots, a_{s-1}, M\right)$ holds.

Proof. We put $A_{j}=a_{j} / G$ and $m=M / G, j=0, \ldots, s-1$. In particular,

$$
\operatorname{gcd}\left(A_{0}, \ldots, A_{s-1}, m\right)=1 .
$$

It is enough to show that the polynomial

$$
H(\mathbf{X})=\sum_{j=0}^{s-1} A_{j}\left(f_{k+j}(\mathbf{X})-f_{l+j}(\mathbf{X})\right)
$$

is nonconstant modulo any prime $p \mid m$, for $k \neq l$. 
By definition, we have

$$
H^{(p)}(\mathbf{X}) \equiv \sum_{j=0}^{s-1} A_{j}\left(f_{k+j}^{(p)}(\mathbf{X})-f_{l+j}^{(p)}(\mathbf{X})\right) \quad(\bmod p)
$$

and $H^{(p)}(\mathbf{X})$ can not be a constant polynomial, since $f^{(p)} \in \mathcal{T}$ and so we are done.

\subsection{Discrepancy}

For a sequence of $N$ points

$$
\Gamma=\left(\gamma_{0, n}, \ldots, \gamma_{s-1, n}\right)_{n=0}^{N-1}
$$

of the half-open interval $[0,1)^{s}$, denote by $\Delta_{\Gamma}$ its discrepancy, that is,

$$
\Delta_{\Gamma}=\sup _{B \subseteq[0,1)^{s}}\left|\frac{T_{\Gamma}(B)}{N}-\right| B||,
$$

where $T_{\Gamma}(B)$ is the number of points of the sequence $\Gamma$ which hit the box

$$
B=\left[\alpha_{0}, \beta_{0}\right) \times \ldots \times\left[\alpha_{s-1}, \beta_{s-1}\right) \subseteq[0,1)^{s}
$$

and the supremum is taken over all such boxes.

For an integer vector $\mathbf{a}=\left(a_{0}, \ldots, a_{s-1}\right) \in \mathbb{Z}^{s}$ we put

$$
|\mathbf{a}|=\max _{i=0, \ldots, s-1}\left|a_{i}\right|, \quad r(\mathbf{a})=\prod_{i=0}^{s-1} \max \left\{\left|a_{i}\right|, 1\right\} .
$$

We need the Erdös-Turán-Koksma inequality (see Theorem 1.21 of [4) for the discrepancy of a sequence of points of the $s$-dimensional unit cube, which we present in the following form.

Lemma 7. There exists a constant $C_{s}>0$ depending only on the dimension $s$ such that, for any integer $L \geq 1$, for the discrepancy of a sequence of points (7) the bound

$$
\Delta_{\Gamma}<C_{s}\left(\frac{1}{L}+\frac{1}{N} \sum_{0<|\mathbf{a}| \leq L} \frac{1}{r(\mathbf{a})}\left|\sum_{n=0}^{N-1} \exp \left(2 \pi i \sum_{j=0}^{s-1} a_{j} \gamma_{j, n}\right)\right|\right)
$$

holds, where $|\mathbf{a}|, r(\mathbf{a})$ are defined by (8) and the sum is taken over all integer vectors

$$
\mathbf{a}=\left(a_{0}, \ldots, a_{s-1}\right) \in \mathbb{Z}^{s}
$$

with $0<|\mathbf{a}| \leq L$.

The currently best value of $C_{s}$ is given in [2]. 


\section{Discrepancy Bound}

Let the sequence $\left(u_{n}\right)$ generated by (1) be purely periodic with an arbitrary period $t$. For an integer vector $\mathbf{a}=\left(a_{0}, \ldots, a_{s-1}\right) \in \mathbb{Z}^{s}$ we introduce the exponential sum

$$
S \mathbf{a}(N)=\sum_{n=0}^{N-1} \mathbf{e}_{M}\left(\sum_{j=0}^{s-1} a_{j} u_{n+j}\right) .
$$

Theorem 1. Let the sequence $\left(u_{n}\right)$, given by (1) with a polynomial $f^{(p)}(\mathbf{X}) \in \mathcal{T}$, for every prime divisor $p$ of $M$, with total degree $d$ and $\operatorname{deg}_{X_{r}} f \geq 1$, be purely periodic with period $t$ and $t \geq N \geq 1$. The bound

$$
\max _{\operatorname{gcd}\left(a_{0}, \ldots, a_{s-1}, M\right)=G}\left|S_{\mathbf{a}}(N)\right|=O\left(N^{1 / 2} M^{r / 2}(\log \log (M / G))^{-1 / 2}\right)
$$

holds, where $G=\operatorname{gcd}\left(a_{0}, \ldots, a_{s-1}, M\right)$ and the implied constant depends only on $s$ and $d$.

Proof. The proof follows a strategy first seen in 9 .

Select any $\mathbf{a}=\left(a_{0}, \ldots, a_{s-1}\right) \in \mathbb{Z}^{s}$ with $\operatorname{gcd}\left(a_{0}, \ldots, a_{s-1}, M\right)=G$.

It is obvious that for any integer $k \geq 0$ we have

$$
\left|S_{\mathbf{a}}(N)-\sum_{n=0}^{N-1} \mathbf{e}_{M}\left(\sum_{j=0}^{s-1} a_{j} u_{n+k+j}\right)\right| \leq 2 k .
$$

Therefore, for any integer $K \geq 1$,

$$
K\left|S_{\mathbf{a}}(N)\right| \leq W+K^{2},
$$

where

$$
W=\left|\sum_{n=0}^{N-1} \sum_{k=0}^{K-1} \mathbf{e}_{M}\left(\sum_{j=0}^{s-1} a_{j} u_{n+k+j}\right)\right| \leq \sum_{n=0}^{N-1}\left|\sum_{k=0}^{K-1} \mathbf{e}_{M}\left(\sum_{j=0}^{s-1} a_{j} u_{n+k+j}\right)\right| .
$$

Accordingly, letting $\mathbf{x}=x_{1}, \ldots, x_{r}$, we obtain

$$
\begin{aligned}
W^{2} & \leq N \sum_{n=0}^{N-1}\left|\sum_{k=0}^{K-1} \mathbf{e}_{M}\left(\sum_{j=0}^{s-1} a_{j} f_{k+j}\left(u_{n}, \ldots, u_{n-r+1}\right)\right)\right|^{2} \\
& \leq N \sum_{\mathbf{x} \in \mathbb{Z}_{M}^{r}}\left|\sum_{k=0}^{K-1} \mathbf{e}_{M}\left(\sum_{j=0}^{s-1} a_{j} f_{k+j}(\mathbf{x})\right)\right|^{2} \\
& =N \sum_{k=0}^{K-1} \sum_{l=0}^{K-1} \sum_{\mathbf{x} \in \mathbb{Z}_{M}^{r}} \mathbf{e}_{M}\left(\sum_{j=0}^{s-1} a_{j}\left(f_{k+j}(\mathbf{x})-f_{l+j}(\mathbf{x})\right)\right) .
\end{aligned}
$$


If $k=l$, then the inner sum is trivially equal to $M^{r}$. There are $K$ such sums. Otherwise the polynomial $\sum_{j=0}^{s-1} a_{j}\left(f_{k+j}(\mathbf{X})-f_{l+j}(\mathbf{X})\right)$ is nonconstant since $f^{(p)} \in \mathcal{T}$. Hence we can apply Lemma 5 and Lemma 6 (so that we only need consider $a_{j}, j=0, \ldots, s-1$, instead of the coefficients of $f$ ) to the inner sum, obtaining the upper bound

$$
e^{c_{0} d^{3(K+s-2)}} M^{r-1 / d^{3(K+s-2)}} G^{1 / d^{3(K+s-2)}}
$$

for at most $K^{2}$ sums and positive constant $c_{0}$ and noting that $d^{2}(\log d+1)<d^{3}$.

Hence,

$$
W^{2} \leq K N M^{r}+K^{2} N e^{c_{0} d^{3(K+s-2)}} M^{r-1 / d^{3(K+s-2)}} G^{1 / d^{3(K+s-2)}} .
$$

Now, without too much loss of generality we may assume $(d+1)^{3(K+s-2)} \geq 2$. Next we put $K=\lceil\log \log (M / G) /(3 c \log (d+1))\rceil$, for some $c>2$ to guarantee that the first term dominates and the result follows.

Next, let $D_{s}(N)$ denote the discrepancy of the points given by

$$
\left(\frac{u_{n}}{M}, \ldots, \frac{u_{n+s-1}}{M}\right), \quad n=0, \ldots, N-1,
$$

in the $s$-dimensional unit cube $[0,1)^{s}$.

Theorem 2. If the sequence $\left(u_{n}\right)$, given by (1) with a polynomial $f^{(p)}(\mathbf{X}) \in \mathcal{T}$, for every prime divisor $p$ of $M$, with total degree $d$ and $\operatorname{deg}_{X_{r}} f \geq 1$ is purely periodic with period $t$ with $t \geq N \geq 1$, then the bound

$$
D_{s}(N)=O\left(N^{-1 / 2} M^{r / 2}(\log \log \log M)^{s} /(\log \log M)^{1 / 2}\right)
$$

holds, where the implied constant depends only on $s$ and $d$.

Proof. The statement follows from Lemma 7, taken with

$$
L=\left\lceil N^{1 / 2} M^{-r / 2}(\log \log M)^{1 / 2}\right\rceil
$$

and the bound of Theorem 11, where all occurring $G=\operatorname{gcd}\left(a_{0}, \ldots, a_{s-1}, M\right)$ are at most $L$

\section{References}

1. Arkhipov, G.I., Chubarikov, V.N., Karatsuba, A.A.: Trigonometric Sums in Number Theory and Analysis, de Gruyter Expositions in Mathematics, Berlin, vol. 39 (2004)

2. Cochrane, T.: Trigonometric approximation and uniform distribution modulo 1. Proc. Amer. Math. Soc. 103, 695-702 (1988)

3. Cochrane, T., Zheng, Z.Y.: A Survey on Pure and Mixed Exponential Sums Modulo Prime Numbers. Proc. Illinois Millenial Conf. on Number Theory 1, 271-300 (2002) 
4. Drmota, M., Tichy, R.F.: Sequences, discrepancies and applications. Springer, Berlin (1997)

5. El-Mahassni, E.D., Shparlinski, I.E., Winterhof, A.: Distribution of nonlinear congruential pseudorandom numbers for almost squarefree integers. Monatsh. Math. 148, 297-307 (2006)

6. El-Mahassni, E.D., Winterhof, A.: On the distribution of nonlinear congruential pseudorandom numbers in residue rings. Intern. J. Number Th. 2(1), 163-168 (2006)

7. Griffin, F., Niederreiter, H., Shparlinski, I.: On the distribution of nonlinear recursive congruential pseudorandom numbers of higher orders. In: Fossorier, M.P.C., Imai, H., Lin, S., Poli, A. (eds.) AAECC 1999. LNCS, vol. 1719, pp. 87-93. Springer, Heidelberg (1999)

8. Gutierrez, J., Gomez-Perez, D.: Iterations of multivariate polynomials and discrepancy of pseudorandom numbers. In: Bozta, S., Sphparlinski, I. (eds.) AAECC 2001. LNCS, vol. 2227, pp. 192-199. Springer, Heidelberg (2001)

9. Niederreiter, H., Shparlinski, I.E.: On the distribution and lattice structure of nonlinear congruential pseudorandom numbers. Finite Fields and Their Appl. 5, 246253 (1999)

10. Niederreiter, H., Shparlinski, I.E.: Exponential sums and the distribution of inversive congruential pseudorandom numbers with prime-power modulus. Acta Arith. 92, 89-98 (2000)

11. Stečkin, S.B.: An estimate of a complete rational exponential sum. Trudy Mat. Inst. Steklov. 143, 188-207 (1977) (in Russian) 